\title{
Interpolation on the sphere: a fast solution technique
}

\author{
T. $\operatorname{Tran}^{1} \quad$ Q. T. Le Gia
}

(Received 11 August 2008; revised 4 November 2008)

Dedicated to Prof. Ian H. Sloan on the occasion of his 70th birthday

\begin{abstract}
We present a fast solution technique for the problem of interpolation on the sphere, using radial basis functions and multiplicative Schwarz methods. This problem has applications in geodesy and earth science. A bound for the condition number of the preconditioned matrix is proved. Since approximation using radial basis functions is a meshless method, the proof technique is novel compared to that used in finite element methods. Numerical experiments on relatively large sets of scattered data points taken from MAGSAT satellite data are presented. The article illustrates how interpolation of scattered data on the sphere can be efficiently performed.
\end{abstract}

http://anziamj.austms.org.au/ojs/index.php/ANZIAMJ/article/view/1412 gives this article, (c) Austral. Mathematical Soc. 2008. Published November 20, 2008. ISSN 1446-8735. (Print two pages per sheet of paper.) 


\section{Contents}

1 Introduction

C355

2 Interpolation by spherical radial basis functions

C357

2.1 Positive definite kernel . . . . . . . . . . . . . . . C357

2.2 Spherical radial basis functions . . . . . . . . . . . . . C359

2.3 Solvability of the interpolation problem . . . . . . . C C359

3 Symmetric multiplicative Schwarz preconditioner $\quad$ C360

3.1 Multiplicative Schwarz operator . . . . . . . . . . . C360

3.2 Algorithm . . . . . . . . . . . . . . . . . . C361

3.3 Subspace decomposition . . . . . . . . . . . . . . C362

3.4 Bound on the condition number of $\mathrm{P}_{\mathrm{smu}} \ldots \ldots \ldots$. . . . . C362

4 Numerical experiments

C364

References

C367

\section{Introduction}

Data interpolation and fitting problems where the underlying domain is the sphere arise in many areas including geodesy and earth science in which the sphere is taken as a model for the earth. Even though in practice measured data usually contain noise, for simplicity we disregard this and consider the interpolation problem instead of the more appropriate approximation problem, with scattered data collected from satellites.

Fasshauer and Schumaker [4] discussed available methods for interpolation of scattered data on the sphere, which use spherical splines [1], and spherical radial basis functions $[9,10]$. We use spherical radial basis functions, a precise definition of which is introduced in Section 2. 
Both theory [7] and numerical experiments (Section 4) show that the matrices arising from this interpolation problem are very ill-conditioned. We present an algorithm which uses spherical radial basis functions to construct the interpolant and a multiplicative Schwarz preconditioner to accelerate the solution process. We give an estimate for the condition number of the preconditioned system.

Even though domain decomposition methods (of which multiplicative Schwarz methods are members) have been extensively studied for finite element and boundary element methods, not much has been done for meshless methods using radial basis functions. For the interpolation problem in $\mathbb{R}^{n}$ using radial basis functions, the idea of dividing the scattered data set into smaller subsets for the purpose of defining the Schwarz alternating algorithm was proposed by Beatson et al. [2]. Work on applying the multiplicative Schwarz alternating algorithm using spherical splines was also carried out by Hesse [5], but in that work the data points are not scattered, and again the Schwarz method is not used as a preconditioner. None of these articles uses domain decomposition methods as preconditioners to be solved with the conjugate gradient method, and none studies the condition numbers of the preconditioned systems. The purpose of this article is to fill this gap. We study in Section 3 the condition number of the system preconditioned by a symmetric multiplicative Schwarz method. Previously [6] we studied the use of additive Schwarz preconditioners for elliptic partial differential equations on the sphere, and proved a bound for the condition number. A similar approach is used in the present article for the interpolation problem. Section 4 also reports our numerical experiments which illustrates the efficiency of the method. 


\section{Interpolation by spherical radial basis functions}

Let $\mathbb{S}$ be the unit sphere in $\mathbb{R}^{3}$, and suppose that $X=\left\{\boldsymbol{x}_{1}, \ldots, \boldsymbol{x}_{\mathrm{N}}\right\}$ is a set of scattered points lying on $\mathbb{S}$. Given real numbers $f_{i}, i=1, \ldots, N$, we want to find a smooth function $\boldsymbol{u}$ defined on $\mathbb{S}$ which interpolates the data, namely,

$$
u\left(x_{i}\right)=f_{i}, \quad i=1, \ldots, N .
$$

The interpolant $\mathfrak{u}(\boldsymbol{x})$ shall be defined in terms of spherical radial basis functions, which in turn are defined from a positive definite kernel on $\mathbb{S}$.

\subsection{Positive definite kernel}

A continuous function $\Phi: \mathbb{S} \times \mathbb{S} \rightarrow \mathbb{R}$ is called a positive definite kernel on $\mathbb{S}$ if it satisfies $\Phi(\boldsymbol{x}, \boldsymbol{y})=\Phi(\mathbf{y}, \boldsymbol{x})$ for all $\boldsymbol{x}, \boldsymbol{y} \in \mathbb{S}$, and if for every set of distinct points $\left\{\boldsymbol{y}_{1}, \ldots, \mathbf{y}_{M}\right\}$ on $\mathbb{S}$, the $\boldsymbol{M} \times \mathbf{M}$ matrix $\boldsymbol{M}$ with entries $\boldsymbol{M}_{i, j}=\Phi\left(\boldsymbol{y}_{i}, \boldsymbol{y}_{j}\right)$ is positive semidefinite. If the matrix $\boldsymbol{M}$ is positive definite, then $\Phi$ is called a strictly positive definite kernel $[11,16]$.

We define the kernel $\Phi$ in terms of a univariate function $\phi:[-1,1] \rightarrow \mathbb{R}$,

$$
\Phi(\boldsymbol{x}, \mathbf{y})=\phi(\boldsymbol{x} \cdot \mathbf{y}) \quad \text { for all } \boldsymbol{x}, \mathbf{y} \in \mathbb{S}
$$

where $\boldsymbol{x} \cdot \boldsymbol{y}$ is the dot product of two vectors $\boldsymbol{x}$ and $\boldsymbol{y}$ on the sphere, which is the cosine of the angle between the two points. If $\phi$ has a series expansion in terms of Legendre polynomials $\mathrm{P}_{\ell}$,

$$
\phi(t)=\frac{1}{4 \pi} \sum_{\ell=0}^{\infty}(2 \ell+1) \hat{\phi}(\ell) P_{\ell}(t),
$$


where $\hat{\phi}(\ell)=2 \pi \int_{-1}^{1} \phi(t) P_{\ell}(t) d t$, then the kernel $\Phi$ is represented as

$$
\Phi(\boldsymbol{x}, \mathbf{y})=\sum_{\ell=0}^{\infty} \hat{\phi}(\ell) \sum_{\mathrm{m}=-\ell}^{\ell} \mathrm{Y}_{\ell, \mathrm{m}}(\boldsymbol{x}) \mathrm{Y}_{\ell, \mathrm{m}}(\mathbf{y})
$$

where $Y_{\ell, m}, m=-\ell, \ldots, \ell$, are spherical harmonics of order $\ell$; see the canonical book by Müller [8].

The kernel $\Phi$ defined with this univariate function $\phi$ is called a zonal kernel. Chen et al. [3] established a complete characterisation of strictly positive definite kernels: the kernel $\Phi$ is strictly positive definite if and only if $\hat{\phi}(\ell) \geq 0$ for all $\ell \geq 0$, and $\hat{\phi}(\ell)>0$ for infinitely many even values of $\ell$ and infinitely many odd values of $\ell$.

We assume that $\hat{\phi}(\ell)>0$ for all $\ell \geq 0$. The native space associated with $\phi$ is defined by

$$
\mathcal{N}_{\phi}:=\left\{v \in \mathcal{D}^{\prime}(\mathbb{S}): \sum_{\ell=0}^{\infty} \sum_{m=-\ell}^{\ell} \frac{\left|\hat{v}_{\ell, m}\right|^{2}}{\hat{\phi}(\ell)}<\infty\right\},
$$

where $\mathcal{D}^{\prime}(\mathbb{S})$ is the space of distributions defined on $\mathbb{S}$, and $\hat{v}_{\ell, m}=\left\langle\nu, Y_{\ell, m}\right\rangle$ with $\langle\cdot, \cdot\rangle$ being the $\mathrm{L}_{2}$-inner product on $\mathbb{S}$. The space $\mathcal{N}_{\phi}$ is equipped with an inner product and a norm defined by

$$
\langle v, w\rangle_{\phi}=\sum_{\ell=0}^{\infty} \sum_{m=-\ell}^{\ell} \frac{\hat{v}_{\ell, m} \hat{w}_{\ell, m}}{\hat{\phi}(\ell)} \text { and }\|v\|_{\phi}=\left(\sum_{\ell=0}^{\infty} \sum_{m=-\ell}^{\ell} \frac{\left|\hat{v}_{\ell, m}\right|^{2}}{\hat{\phi}(\ell)}\right)^{1 / 2} .
$$

If the coefficients $\hat{\phi}(\ell)$ for $\ell=0,1, \ldots$ satisfy

$$
c_{1}(\ell+1)^{-2 \tau} \leq \hat{\phi}(\ell) \leq c_{2}(\ell+1)^{-2 \tau}
$$

for some positive constants $c_{1}$ and $c_{2}$, and some $\tau \in \mathbb{R}$, then the native space $\mathcal{N}_{\phi}$ is identified with the Sobolev space $\mathrm{H}^{\tau}(\mathbb{S})$, and the corresponding norms are equivalent. In particular, if $\tau>1$ then the series (3) converges pointwise and $\mathcal{N}_{\phi} \subset \mathrm{C}(\mathbb{S})$, which is essentially the Sobolev embedding theorem. Here $C(\mathbb{S})$ is the space of continuous functions on $\mathbb{S}$. 


\subsection{Spherical radial basis functions}

Recall that $X=\left\{\boldsymbol{x}_{1}, \ldots, \boldsymbol{x}_{\mathrm{N}}\right\}$ is the set of interpolating points on the sphere. The spherical radial basis functions $\Phi_{j}, j=1, \ldots, N$, associated with $\mathrm{X}$ and the kernel $\Phi$ are defined by

$$
\Phi_{j}(\boldsymbol{x}):=\Phi\left(\boldsymbol{x}, \boldsymbol{x}_{\mathfrak{j}}\right)=\sum_{\ell=0}^{\infty} \sum_{\mathrm{m}=-\ell}^{\ell} \hat{\phi}(\ell) \mathrm{Y}_{\ell, \mathrm{m}}\left(\boldsymbol{x}_{\mathfrak{j}}\right) \mathrm{Y}_{\ell, \mathrm{m}}(\boldsymbol{x}) .
$$

We note that, for any continuous function $v$ defined on the sphere,

$$
v\left(\boldsymbol{x}_{\mathbf{j}}\right)=\sum_{\ell=0}^{\infty} \sum_{\mathrm{m}=-\ell}^{\ell} \frac{\hat{v}_{\ell, \mathrm{m}} \hat{\phi}(\ell) \mathrm{Y}_{\ell, \mathrm{m}}\left(\boldsymbol{x}_{\mathbf{j}}\right)}{\hat{\phi}(\ell)}=\left\langle v, \Phi_{\mathbf{j}}\right\rangle_{\phi}, \quad j=1, \ldots, \mathrm{N} .
$$

In other words, $\Phi$ is the reproducing kernel of the native space $\mathcal{N}_{\phi}$ when $\tau>1$.

Let $\mathrm{V}:=\mathrm{V}_{\mathrm{X}}^{\phi}:=\operatorname{span}\left\{\Phi_{1}, \ldots, \Phi_{\mathrm{N}}\right\}$. We note that if $\tau>1$, then $\mathrm{V} \subset$ $\mathcal{N}_{\phi}=\mathrm{H}^{\tau}(\mathbb{S}) \subset \mathrm{C}(\mathbb{S})$. The interpolant $u$ satisfying (1) is sought in this finite dimensional space $\mathrm{V}$.

\subsection{Solvability of the interpolation problem}

The interpolant $u \in V$ is uniquely defined thanks to the positive definiteness of the kernel $\Phi$. Indeed, by writing $u=\sum_{j=1}^{N} c_{j} \Phi_{j}$ we rewrite (1) as

$$
\sum_{j=1}^{N} c_{j} \Phi_{j}\left(x_{i}\right)=f_{i}, \quad i=1, \ldots, N
$$

or in matrix form

$$
A \mathbf{c}=\mathbf{f}
$$


where $\mathbf{A}=\left(\Phi_{j}\left(\boldsymbol{x}_{i}\right)\right)_{i, j=1}^{\mathbf{N}}, \mathbf{c}=\left(\mathbf{c}_{j}\right)_{j=1}^{\mathrm{N}}$, and $\mathbf{f}=\left(\boldsymbol{f}_{j}\right)_{j=1}^{\mathrm{N}}$. The matrix $\mathbf{A}$ is both symmetric and positive definite (see Sections 2.1 and 2.2), and so (6) is solved by using, for example, the conjugate gradient method.

Due to (5), equation (1) is rewritten as

$$
\left\langle u, \Phi_{i}\right\rangle_{\phi}=\left\langle f, \Phi_{i}\right\rangle_{\phi}, \quad i=1, \ldots, N,
$$

where $f$ is a smooth function satisfying $f\left(x_{i}\right)=f_{i}$. We note that the function $f$ is introduced here purely for analysis purposes.

Error analysis for this problem was carried out by Narcowich et al. [9, 10]. Levesley et al. [7] to show that the matrix $\boldsymbol{A}$ is ill-conditioned. In the following section, we design a preconditioner for this system, using multiplicative Schwarz methods.

\section{Symmetric multiplicative Schwarz preconditioner}

\subsection{Multiplicative Schwarz operator}

Thanks to (7) we view the interpolation problem (1) as a variational problem, with a bilinear form defined on $\mathrm{H}^{\tau}(\mathbb{S})$ by

$$
a(v, w):=\langle v, w\rangle_{\phi} \quad \text { for all } v, w \in \mathrm{H}^{\tau}(\mathbb{S}) .
$$

By using this bilinear form, we define the additive and multiplicative Schwarz operators as usual $[12,13]$.

Definition 1 Let $\mathrm{J}$ be a positive integer. Let $\mathrm{V}$ be decomposed as $\mathrm{V}=\mathrm{V}_{0}+$ $\cdots+\mathrm{V}_{\mathrm{J}}$, where $\mathrm{V}_{\mathrm{k}}, \mathrm{k}=0, \ldots, \mathrm{J}$, are subspaces of $\mathrm{V}$. 
1. The projections $\mathrm{P}_{\mathrm{k}}: \mathrm{V} \rightarrow \mathrm{V}_{\mathrm{k}}, \mathrm{k}=0, \ldots, \mathrm{J}$, are defined by

$$
\mathrm{a}\left(\mathrm{P}_{\mathrm{k}} v, w\right)=\mathrm{a}(v, w) \quad \text { for all } v \in \mathrm{V}, w \in \mathrm{V}_{\mathrm{k}}
$$

2. The additive Schwarz operator is defined by $\mathrm{P}_{a d}=\mathrm{P}_{0}+\cdots+\mathrm{P}_{\mathrm{J}}$.

3. The symmetric multiplicative Schwarz operator is defined by $\mathrm{P}_{s m u}=$ $\mathrm{I}-\mathrm{E}_{m u}^{\mathrm{t}} \mathrm{E}_{m u}$, where $\mathrm{I}$ is the identity operator on $\mathrm{V}, \mathrm{E}_{m u}$ is the error propagation operator defined by $\mathrm{E}_{m u}=\left(\mathrm{I}-\mathrm{P}_{\mathrm{J}}\right) \cdots\left(\mathrm{I}-\mathrm{P}_{\mathrm{o}}\right)$, and $\mathrm{E}_{m u}^{\mathrm{t}}$ is its transpose.

The operator $\mathrm{P}_{\mathrm{ad}}$ is introduced here purely for the analysis purpose; see the proof of Theorem 2. In the abstract form, solving (6) by a symmetric multiplicative Schwarz method is solving $P_{\text {smu }} u=g$, where $g$ is obtained from $f$ by solving subproblems; see the book by Toselli and Widlund [13] for more details. In the implementation, one solves (6) by the preconditioned conjugate gradient method, which requires an algorithm to compute the action of the preconditioner $\boldsymbol{A}_{\mathrm{smu}}^{-1}$ on a residual vector $\boldsymbol{r}$, where $\boldsymbol{A}_{\mathrm{smu}}^{-1}$ is related to the matrix representation $\mathbf{P}_{\mathrm{smu}}$ of $\mathrm{P}_{\mathrm{smu}}$ by $\mathbf{P}_{\mathrm{smu}}=\boldsymbol{A}_{\mathrm{smu}}^{-1} \boldsymbol{A}$. This algorithm is defined in the following subsection.

\subsection{Algorithm}

For $k=0, \ldots, J$, let $\boldsymbol{A}_{k}$ be the restriction of the matrix $\boldsymbol{A}$ onto each subspace $V_{k}$, and let $\boldsymbol{R}_{k}$ be the matrix that transforms the basis of $V$ to the basis of $\boldsymbol{V}_{k}$. Then for any $\mathbf{r} \in \mathbb{R}^{\mathrm{N}}$, Algorithm 1 computes $\boldsymbol{A}_{\text {smu }}^{-1} \mathbf{r}$.

The key point is how to define the subspace decomposition $\mathrm{V}=\mathrm{V}_{\mathrm{O}}+$ $\cdots+V_{J}$, which must be different from finite element or boundary element methods. These methods define subdomains from a partition (or mesh) of the geometry into elements. In the present case, there is no mesh, and thus the subdomains will be defined based on scattered data. 


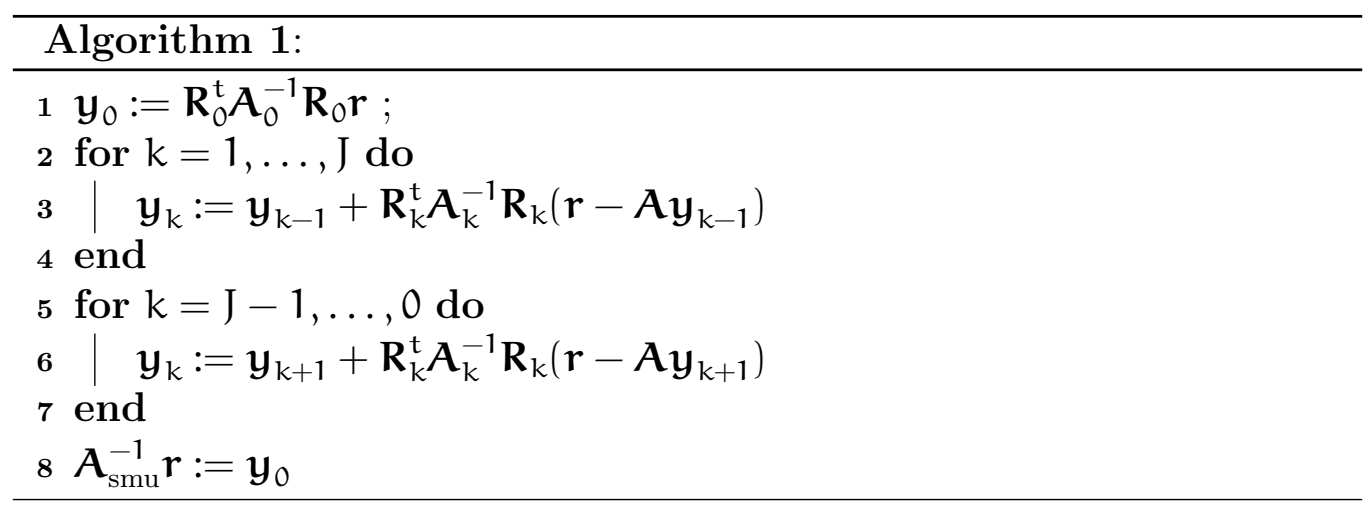

\subsection{Subspace decomposition}

To define the subspace decomposition, we first decompose the data set $X$ in the form $X=X_{0} \cup \cdots \cup X_{J}$ as in Algorithm 2.

The reason to include the condition on $\alpha$ in Step (8)-(9) is to ensure that the centres are not clustered at one place which may result in a situation in which not all points in $X$ are covered. The subsets $X_{k}$ overlap. The subspaces $V_{k}$ are defined by

$$
V_{k}=\operatorname{span}\left\{\Phi_{j}: x_{j} \in X_{k}\right\}, \quad k=0, \ldots, J .
$$

The set $X_{0}$ defining $V_{0}$ is analogous to the coarse grid in finite element methods, which is necessary for global communication, as explained by Toselli and Widlund [13].

\subsection{Bound on the condition number of $P_{\text {smu }}$}

We now state and sketch the proof of our main result. 


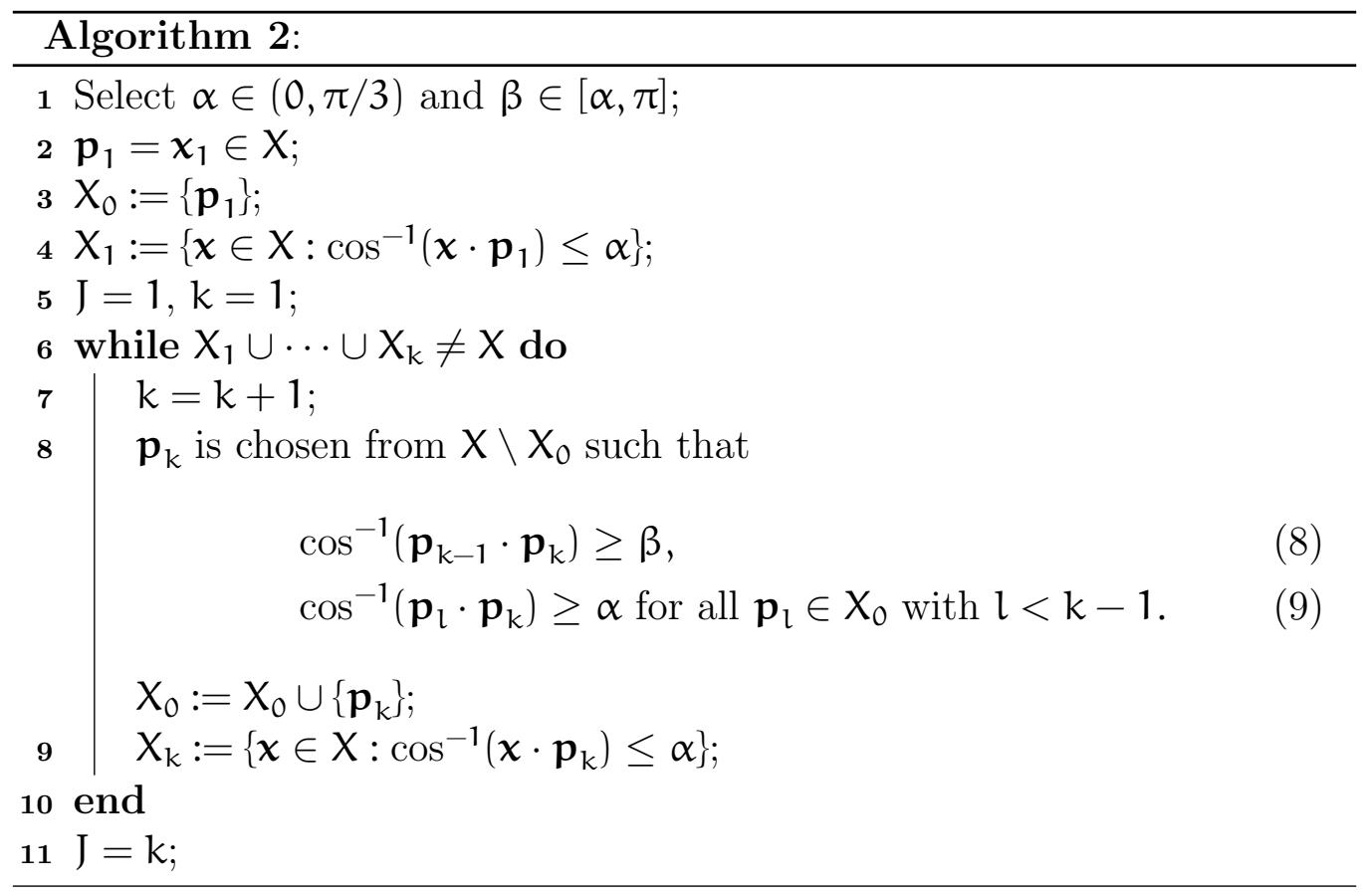


Theorem 2 The extremum eigenvalues of $\mathrm{P}_{\text {smu }}$ are bounded by

$$
\lambda_{\max }\left(\mathrm{P}_{s m u}\right) \leq 1 \quad \text { and } \quad \lambda_{\min }\left(\mathrm{P}_{s m u}\right) \geq \frac{1}{\left(1+2 \mathrm{~J}^{2}\right)\left(1+\mathrm{J}\left(1-\|\tilde{\mathrm{Q}}\|_{\phi}\right)^{-2}\right)},
$$

so that

$$
\kappa\left(\mathrm{P}_{s m u}\right) \leq\left(1+2 \mathrm{~J}^{2}\right)\left(1+\frac{\mathrm{J}}{\left(1-\|\tilde{\mathrm{Q}}\|_{\phi}\right)^{2}}\right),
$$

where $\|\tilde{\mathrm{Q}}\|_{\phi}$ is the operator norm of $\tilde{\mathrm{Q}}:=\left(\mathrm{I}-\mathrm{P}_{\mathrm{J}}\right) \cdots\left(\mathrm{I}-\mathrm{P}_{1}\right)$ defined from the norm of the native space $\mathcal{N}_{\phi}$.

Proof: The bound for $\lambda_{\max }\left(\mathrm{P}_{\mathrm{smu}}\right)$ is standard as described by Toselli and Widlund [13]. We prove, using the same technique as in our previous articles $[6,14]$, that $\lambda_{\min }\left(P_{\mathrm{ad}}\right) \geq 1 /\left[1+\mathrm{J}\left(1-\|\tilde{Q}\|_{\phi}\right)^{-2}\right]$. The bound for $\lambda_{\min }\left(\mathrm{P}_{\mathrm{smu}}\right)$ then follows by using standard arguments for Schwarz operators; see the book by Toselli and Widlund [13].

Our numerical experiments show that $\|\tilde{Q}\|_{\phi}$ depends on $\alpha$ and $\beta$; see previous works for more discussion $[6,14]$. The theoretical bound is not sharp, and further study is in progress.

\section{$4 \quad$ Numerical experiments}

In the experiments, in order to check the accuracy of our method, we chose to interpolate a known function, namely, $f(\boldsymbol{x})=\exp \left(x_{1}+x_{2}+x_{3}\right)$, where $\boldsymbol{x}=\left(x_{1}, x_{2}, x_{3}\right) \in \mathbb{S}$. The univariate function $\phi$ defining the kernel $\Phi$, see (2), is defined by $\phi(t)=\rho_{m}(\sqrt{2-2 t})$, where $\rho_{m}$ are Wendland's functions [15] given in Table 1. Narcowich and Ward prove [10, Proposition 4.6] that (4) holds with $\tau=m+3 / 2$. 
TABLE 1: Wendland's radial basis functions

\begin{tabular}{|c|l|c|}
\hline $\mathrm{m}$ & \multicolumn{1}{|c|}{$\rho_{\mathrm{m}}(r)$} & $\tau$ \\
\hline 1 & $(1-r)_{+}^{4}(4 r+1)$ & 2.5 \\
2 & $(1-r)_{+}^{6}\left(35 r^{2}+18 r+3\right)$ & 3.5 \\
3 & $(1-r)_{+}^{8}\left(32 r^{3}+25 r^{2}+8 r+1\right)$ & 4.5 \\
\hline
\end{tabular}

TABLE 2: Unpreconditioned systems

\begin{tabular}{|cc|ccc|rr|}
\hline $\mathrm{m}$ & $\mathrm{N}$ & $\lambda_{\min }$ & $\lambda_{\max }$ & $\kappa$ & $\mathrm{CPU}$ & iter \\
\hline 1 & 12345 & $0.3241 \mathrm{e}-03$ & $0.4966 \mathrm{e}+03$ & $0.1532 \mathrm{e}+07$ & 3159 & 1321 \\
& 24689 & $0.5352 \mathrm{e}-03$ & $0.9942 \mathrm{e}+03$ & $0.1858 \mathrm{e}+07$ & 16703 & 1362 \\
& 49377 & $0.2093 \mathrm{e}-02$ & $0.1988 \mathrm{e}+04$ & $0.9496 \mathrm{e}+06$ & 55082 & 994 \\
\hline 2 & 12345 & $0.1448 \mathrm{e}-02$ & $0.1198 \mathrm{e}+04$ & $0.8275 \mathrm{e}+06$ & 2379 & 985 \\
& 24689 & $0.3875 \mathrm{e}-02$ & $0.2400 \mathrm{e}+04$ & $0.6193 \mathrm{e}+06$ & 11350 & 880 \\
& 49377 & $0.1257 \mathrm{e}-01$ & $0.4799 \mathrm{e}+04$ & $0.3816 \mathrm{e}+06$ & 48747 & 740 \\
\hline 3 & 12345 & $0.1417 \mathrm{e}-02$ & $0.3315 \mathrm{e}+03$ & $0.2339 \mathrm{e}+06$ & 2206 & 642 \\
& 24689 & $0.2977 \mathrm{e}-02$ & $0.6639 \mathrm{e}+03$ & $0.2230 \mathrm{e}+06$ & 6620 & 639 \\
& 49377 & $0.6010 \mathrm{e}-02$ & $0.1328 \mathrm{e}+04$ & $0.2209 \mathrm{e}+06$ & 43617 & 638 \\
\hline
\end{tabular}


TABle 3: Preconditioned systems

\begin{tabular}{|cc|ccc|ccc|rr|}
\hline $\mathrm{m}$ & $\mathrm{N}$ & $\cos \alpha$ & $\cos \beta$ & $\mathrm{J}$ & $\lambda_{\min }$ & $\lambda_{\max }$ & $\mathrm{K}$ & $\mathrm{CPU}$ & iter \\
\hline 1 & 12345 & 0.57 & -0.66 & 11 & $9.9855 \mathrm{e}-01$ & 1.0 & 1.000 & 334 & 2 \\
& 12345 & 0.55 & -0.63 & 11 & $9.9915 \mathrm{e}-01$ & 1.0 & 1.000 & 374 & 2 \\
& 24689 & 0.80 & -0.77 & 25 & $9.9657 \mathrm{e}-01$ & 1.0 & 1.003 & 1858 & 2 \\
& 24689 & 0.70 & -0.86 & 16 & $9.9817 \mathrm{e}-01$ & 1.0 & 1.002 & 2017 & 2 \\
& 49377 & 0.95 & -0.49 & 94 & $9.4714 \mathrm{e}-01$ & 1.0 & 1.056 & 22401 & 2 \\
& 49377 & 0.90 & -0.57 & 49 & $9.8726 \mathrm{e}-01$ & 1.0 & 1.013 & 12082 & 2 \\
\hline 2 & 12345 & 0.57 & -0.66 & 11 & $9.8318 \mathrm{e}-01$ & 1.0 & 1.017 & 334 & 2 \\
& 12345 & 0.55 & -0.63 & 11 & $9.9983 \mathrm{e}-01$ & 1.0 & 1.000 & 374 & 2 \\
& 24689 & 0.80 & -0.77 & 25 & $9.5922 \mathrm{e}-01$ & 1.0 & 1.043 & 1922 & 2 \\
& 24689 & 0.70 & -0.86 & 16 & $9.9962 \mathrm{e}-01$ & 1.0 & 1.000 & 2075 & 2 \\
& 49377 & 0.95 & -0.49 & 94 & $6.8766 \mathrm{e}-01$ & 1.0 & 1.454 & 32542 & 3 \\
& 49377 & 0.90 & -0.57 & 49 & $5.0194 \mathrm{e}-01$ & 1.0 & 1.992 & 18526 & 3 \\
\hline 3 & 12345 & 0.57 & -0.66 & 11 & $9.5568 \mathrm{e}-01$ & 1.0 & 1.046 & 452 & 2 \\
& 12345 & 0.55 & -0.63 & 11 & $9.9957 \mathrm{e}-01$ & 1.0 & 1.000 & 505 & 2 \\
& 24689 & 0.80 & -0.77 & 25 & $9.3008 \mathrm{e}-01$ & 1.0 & 1.075 & 1512 & 2 \\
& 24689 & 0.70 & -0.86 & 16 & $9.9991 \mathrm{e}-01$ & 1.0 & 1.000 & 1610 & 2 \\
& 49377 & 0.95 & -0.49 & 94 & $4.4342 \mathrm{e}-01$ & 1.0 & 2.255 & 49555 & 4 \\
& 49377 & 0.90 & -0.57 & 49 & $3.1444 \mathrm{e}-01$ & 1.0 & 3.180 & 27771 & 4 \\
\hline
\end{tabular}


We solved the problem using various data sets $X$ extracted from a very large set $\mathcal{X}$ of $29,672,661$ data points collected by NASA satellite MAGSAT. These sets are defined as follows. First, we used a thinning process [14] to extract from $\mathcal{X}$ a set $X$ satisfying $\mathrm{q}_{\mathrm{X}}=\pi / 600$, where $\mathrm{q}_{\mathrm{X}}$ is the separation radius defined by

$$
\mathrm{q}_{X}:=\frac{1}{2} \min _{\substack{i, j \in\{1, \ldots, N\} \\ i \neq j}} \cos ^{-1}\left(\boldsymbol{x}_{i} \cdot \boldsymbol{x}_{j}\right) .
$$

Here $N=49,377$ is the cardinality of $X$, which is not pre-determined but is a consequence of the thinning process. This set $X$ which covers the whole sphere is not necessarily the unique set having separation radius $q_{x}=\pi / 600$. In order to compare the condition numbers, we also solved the problem on smaller subsets of $X$ (by successively removing points of even indices). This results in sets of cardinalities 24,689 and 12,345. (Results for smaller sets are available but are not included in the article due to page limitation.)

The results for the unpreconditioned matrices are presented in Table 2, and for the preconditioned matrices in Table 3, with various values of $\mathrm{m}$, namely $m=1,2,3$. This table clearly shows that $\lambda_{\max }\left(P_{\text {smu }}\right)$ is bounded by 1 . A clear advantage of the preconditioner is observed, both in terms of condition numbers $\mathrm{K}$, CPU times, and number of iterations (Iter).

Acknowledgements The second author is supported by the Australian Research Council under its Centres of Excellence program.

\section{References}

[1] P. Alfeld, M. Neamtu, and L. L. Schumaker. Fitting scattered data on sphere-like surfaces using spherical splines. J. Comput. Appl. Math., 73:5-43, 1996. doi:10.1016/0377-0427(96)00034-9. C355 
[2] R. K. Beatson, W. A. Light, and S. Billings. Fast solution of the radial basis function interpolation equations: domain decomposition method. SIAM J. Sci. Comput., 22:1717-1740, 2000. doi:10.1137/S1064827599361771. C356

[3] D. Chen, V. A. Menegatto, and X. Sun. A necessary and sufficient condition for strictly positive definite functions on spheres. Proc. Amer. Math. Soc., 131:2733-2740, 2003. doi:10.1090/S0002-9939-03-06730-3. C358

[4] G. E. Fasshauer and L. L. Schumaker. Scattered data fitting on the sphere. In M. Dahlen, T. Lyche, and L. L. Schumaker, editors, Mathematical Methods for Curves and Surfaces II, pages 117-166, Nashville, 1998. Vanderbilt University Press. C355

[5] K. Hesse. Domain Decomposition Methods in Multiscale Geopotential Determination from SST and $S G G$. PhD thesis, University of Kaiserslautern, Germany, 2002. C356

[6] Q. T. Le Gia, I. H. Sloan, and T. Tran. Overlapping additive Schwarz preconditioners for elliptic PDEs on the unit sphere. Math. Comp., 78:79-101, 2009. doi:10.1090/S0025-5718-08-02150-9. C356, C364

[7] J. Levesley, Z. Luo, and X. Sun. Norm estimates of interpolation matrices and their inverses associated with strictly positive definite functions. Proc. Amer. Math. Soc., 127:2127-2134, 1999. doi:10.1090/S0002-9939-99-04683-3. C356, C360

[8] C. Müller. Spherical Harmonics, volume 17 of Lecture Notes in Mathematics. Springer-Verlag, Berlin, 1966. C358

[9] F. J. Narcowich, X. Sun, J. D. Ward, and H. Wendland. Direct and inverse Sobolev error estimates for scattered data interpolation via spherical basis functions. Found. Comput. Math., 7:369-390, 2007. doi:10.1007/s10208-005-0197-7. C355, C360 
[10] F. J. Narcowich and J. D. Ward. Scattered data interpolation on spheres: error estimates and locally supported basis functions. SIAM J. Math. Anal., 33:1393-1410, 2002. doi:10.1137/S0036141001395054. C355, C360, C364

[11] I. J. Schoenberg. Positive definite function on spheres. Duke Math. J., 9:96-108, 1942. doi:10.1215/S0012-7094-42-00908-6. C357

[12] B. Smith, P. Bjørstad, and W. Gropp. Domain Decomposition-Parallel Multilevel Methods for Elliptic Partial Differential Equations. Cambridge University Press, Cambridge, 1996. C360

[13] A. Toselli and O. Widlund. Domain Decomposition Methods-Algorithms and Theory, volume 34 of Springer Series in Computational Mathematics. Springer-Verlag, Berlin, 2005. C360, C361, C362, C364

[14] T. Tran, Q. T. Le Gia, and I. H. Sloan. Overlapping additive Schwarz preconditioners for interpolation on the unit sphere by spherical radial basis functions. In preparation.

http://www.maths. unsw.edu.au/ qlegia/interp_pcg3.pdf. C364, C367

[15] H. Wendland. Scattered Data Approximation. Cambridge University Press, Cambridge, 2005. C364

[16] Y. Xu and E. W. Cheney. Strictly positive definite functions on spheres. Proc. Amer. Math. Soc., 116:977-981, 1992. doi:10.2307/2159477. C357

\section{Author addresses}

1. T. Tran, School of Mathematics and Statistics, University of New South Wales, Sydney 2052, Australia. 
mailto: thanh.tran@unsw.edu.au

2. Q. T. Le Gia, School of Mathematics and Statistics, University of New South Wales, Sydney 2052, Australia.

mailto:qlegia@unsw.edu.au 